\title{
Dielectric Loaded Impedance Matching for Wideband Implanted Antennas
}

\author{
Tharaka Dissanayake, Member, IEEE, Karu P. Esselle, Senior Member, IEEE, and Mehmet R. Yuce, Member, IEEE
}

\begin{abstract}
In implanted biomedical devices, due to the presence of surrounding dissipative biological tissue, the antenna suffers poor impedance matching. This causes degradation in the performance of a wideband or ultra-wideband (UWB) implanted device. Moreover, the electrical properties of tissue change from organ to organ, and possibly from time to time. In this paper, it is shown that loading of antennas with suitable insulators can deliver broadband matching across a range of dissipative medium properties. An impedance-matched UWB antenna designed to operate inside a lossy medium, which has varying electromagnetic properties within the range expected in biological tissues, is presented. The operating bandwidth of the proposed design is $3.5-4.5 \mathrm{GHz}$, which is an interference-free subset of the unlicensed UWB band in the US. It is demonstrated that once the dielectric loading is applied, the conventional procedure for antenna design in free space can be followed. The proposed implantable small capsule-shaped slot antenna has been characterized using numerical simulations. Details of a proof-of-concept experiment are presented.
\end{abstract}

Index Terms-Biomedical electronic, biomedical engineering, implanted device, ultra-wideband (UWB) antenna.

\section{INTRODUCTION}

A NTENNAS intended for biomedical implants have strict size constraints and they are required to operate in highly dissipative and dense media. Those include muscle, skin [1], brain [2], and delicate locations such as retina [3]. In applications of which the in-body communication device in implanted, such as wireless body area networks [4] or medication monitoring [5], the surrounding environment of the antenna depends on the location of the implant. Even when the implant is fixed, the tissue properties can change due to physiological reasons, from person to person or from time to time [6]. Consequently, the antennas for many implanted devices have to operate in lossy surrounding media with variable electrical properties. In addition to propagation losses [7], [8], key antenna characteristics such as impedance matching, and hence, performance, can vary as a result of tissue property variations. For those biomedical implanted devices with stringent power constraints, an unmatched antenna is not desirable at all. It degrades the quality of communications,

Manuscript received February 20, 2009; revised July 15, 2009. First published September 09, 2009; current version published October 14, 2009.

T. Dissanayake and M. R. Yuce are with the School of Electrical Engineering and Computer Science, University of Newcastle, Callaghan, N.S.W. 2308, Australia (e-mail: tharaka.dissanayake@ newcastle.edu.au).

K. P. Esselle is with the Centre for Wireless Applications, Electronics Engineering, Macquarie University, Sydney, N.S.W. 2109, Australia (e-mail: karu. esselle@mq.edu.au).

Color versions of one or more of the figures in this paper are available online at http://ieeexplore.ieee.org.

Digital Object Identifier 10.1109/TMTT.2009.2029664 reduces the overall efficiency of the radio system, and shortens the battery life of the device, and moreover, the signal levels at the receiver. Previously reported implanted antennas have used superstrates and substrates with dielectric constant in the range of 3-10 to achieve acceptable impedance matching [1], [2], [9], [10]. In [11], a silicone superstrate makes the antenna biocompatible as well. It has been shown that the thickness and the electromagnetic properties of superstrates affect impedance matching, as well as miniaturization of the antenna [12]. In this paper, we focus on wideband body area network applications as defined by IEEE802.15 (under TG6) standards. The ultimate goal of the theoretical and experimental research presented here is to achieve a low-power high-data-rate implantable wireless system. Such systems will support higher sampling rates and measurement of multiple physiological parameters over multiple channels [13]. If implanted, system proposed in [13] needs an antenna well matched inside a highly dissipative medium. A well-matched antenna under all possible conditions results in low power consumption of the radio system, and hence, longer battery life. In our previous study [14], we have considered antennas based on an electric dipole principle and shown that their performance can be severely affected by the presence of tissue material. This is because planar ultra-wideband (UWB) dipoles have strong edge currents and strong electric fields near the tapered feed gap. Nearby tissues disturb these currents and near-field electric fields, resulting in significant changes to antenna characteristics. In contrast, UWB antennas based on the magnetic dipole principle, including wide slot antennas presented in [15] for different types of applications, are less perturbed by near-field objects [16]. Hence, in this research, a slot antenna has been investigated to achieve wideband impedance matching inside a highly dissipative medium.

In this paper, we first investigate the behavior of insulated dipoles to determine the most appropriate insulation permittivity for a dielectric loaded UWB antenna suitable for implanted biomedical applications. We approach the problem by investigating the behavior of the reflection coefficient at the insulator-tissue interface based on analytical expressions derived in [17]. Varying permittivity and conductivity of both the insulating material and the surrounding dissipative medium is considered. Conductivities and permittivities of dissipative media, namely, tissue and fluids of different organs, from [18], will be used in this analysis and later in simulations. By applying the theory that an antenna can be represented as a collection of infinitesimal dipoles [19] and based on the knowledge achieved in the reflection analysis, a miniature capsule-shaped UWB slot antenna, loaded with suitable insulating material, is proposed. 


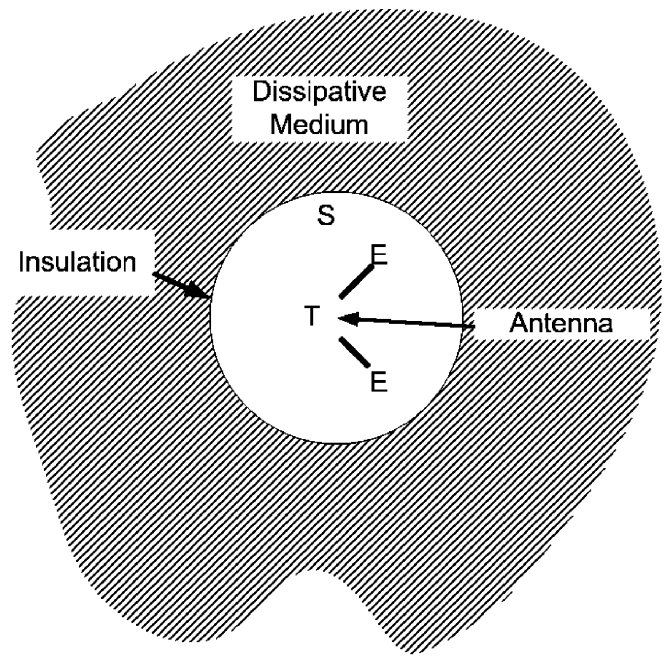

Fig. 1. Insulated antenna in a dissipative medium.

\section{THEORY}

\section{A. Dipoles in Dissipative Medium}

Consider a radiating antenna enclosed in an insulating material, as shown in Fig. 1. The insulated antenna is immersed in a dissipative medium representing biological tissue. Time-domain transient analysis of such an antenna, when radiating a pulse, readily identifies several points of discontinuities where reflection of the incident waves occurs [20]. In the free-space scenario, the most prominent are the reflections at the feed point $(T)$ and ends $(E)$. In general, $E$ can be any discontinuity in the current path apart from the feed point. For example, in a half-wave linear dipole antenna, there are two ends of reflections $(E)$. An explanation of the transient effects of end reflections of a thin wire antenna is given in [21]. In case of implanted antennas, when the boundary between insulation and the external medium is in the proximity, reflections at the surface $(S)$ also contribute to the reflected wave as observed at the feed point $(T)$. A strong reflected wave as seen at the feed point means higher values of $\left|S_{11}\right|$ in the frequency domain. In general, the bandwidth enhancement of pulse radiating antennas has been achieved by minimizing the reflected wave observed at the feed point. Applying resistive coatings at the end points $(E)$ to subside the reflected wave is one such method to reduce internal antenna reflections from ends $(E)$. This method is not applicable to implanted antennas as it reduces the efficiency significantly. For antennas operating in dissipative medium, the reflections at $S$ are also prominent and their minimization, together with other artefacts of the reflected wave, results in wideband impedance matching. Hence, we shall now investigate the behavior of the reflection coefficient at $S$, based on the theoretical expression given in [17].

Consider a Hertzian dipole in the center of a sphere of radius $R$ filled with insulating material with relative permittivity $\varepsilon_{1}$. It is surrounded by a dissipative medium characterized by relative permittivity $\varepsilon_{2}$ and conductivity $\sigma_{2}$. Permeability of each media is $\mu_{0}$, equal to that of free space and tissue material. The complex reflection coefficient of the Gaussian potential, $\Gamma$, at the interface between insulation and dissipative media, $S$, is given by [17, eq. (22)]

$$
\Gamma=\frac{-\left(k_{1}-k_{2}\right)\left[\left(k_{1}+k_{2}\right)\left(1+j k_{1} R\right)\left(1+j k_{2} r\right)-j k_{1}^{2} k_{2}^{2} R^{3}\right]}{\left(k_{1}+k_{2}\right)\left[\left(k_{1}-k_{2}\right)\left(1-j k_{1} R\right)\left(1+j k_{2} R\right)+j k_{1}^{2} k_{2}^{2} R^{3}\right]}
$$

where

$$
k_{1}=\omega \sqrt{\varepsilon_{1} \varepsilon_{o} \mu_{0}}
$$

and

$$
\begin{aligned}
k_{2}=\omega \sqrt{\frac{\varepsilon_{2} \varepsilon_{0} \mu_{0}}{2}} & \left(\sqrt{1+\frac{\sigma_{2}^{2}}{\varepsilon_{2}^{2} \varepsilon_{0}^{2} \omega^{2}}}+1\right)^{1 / 2} \\
& -j \omega \sqrt{\frac{\varepsilon_{2} \varepsilon_{0} \mu_{0}}{2}}\left(\sqrt{1+\frac{\sigma_{2}^{2}}{\varepsilon_{2}^{2} \varepsilon_{0}^{2} \omega^{2}}}-1\right)^{1 / 2}
\end{aligned}
$$

where $k_{1}$ and $k_{2}$ are the propagation constants in each medium. Note that $|\Gamma|^{2}$ is the power reflection coefficient at the interface $(S)$. Obviously, $\Gamma$ is a function of permittivity and conductivity of each medium. To investigate its behavior, a parametric study may be conducted across four different variables $\left(\varepsilon_{1}, R, \varepsilon_{2}, \sigma_{2}\right)$ at a given frequency. However, a normalized (electrical) distance $D=k_{1} R$ can be used to jointly represent two of those ( $\varepsilon_{1}$ and $R$ ) and the two properties of dissipative medium ( $\varepsilon_{2}$ and $\sigma_{2}$ ) shall be used as the second and third independent variables.

Shown in Fig. 2 is the behavior of $\Gamma$ with $\varepsilon_{2}$ and $\sigma_{2}$ for four different values of $D=k_{1} R$. $D$ is varied by varying $R$ while the frequency (set to $4 \mathrm{GHz}$ ) and $\varepsilon_{1}$ (set to 50 ) remain constants. It can be immediately noticed that the minima of $\Gamma$ occur at $\varepsilon_{2}=50$ and $\sigma_{2} \approx 0$. This is quite expected since under those conditions the two media matches perfectly with each other. They are indeed identical electromagnetically; hence, the discontinuity otherwise felt by the outward propagating wave is nonexistent in this special case.

As we move away from this point, the nature of the variation of $\Gamma$ is dependent on the value of electrical distance $D$. As can be seen in Fig. 2(a), for a smaller value of $D=0.1$, reflection changes rapidly with both $\varepsilon_{2}$ and $\sigma_{2}$. Also note that minimum value of $\Gamma>0.8$. This is not favorable if maximum outward energy transmission is intended. In contrast, when $D$ is gradually increased toward a value of $5, \Gamma$ becomes less dependent on properties of the dissipative medium. As shown in Fig. 2(d), it remains less than 0.1 over the range of $\sigma_{2}$ from 0 to $4 \mathrm{~S} / \mathrm{m}$. This distance gives close to zero reflection coefficient over a wide range of $\varepsilon_{2}$. Therefore, we can conclude that, at a given frequency and $\varepsilon_{1}$, higher $D$ leads to lower reflections at the insulator boundary across a wide range of dissipative medium properties.

To generalize the above conclusion for other frequencies and dielectric constants of the insulating medium, let us further investigate the relationship in (2). It is clear that $k_{1}$ is a function of frequency and permittivity. Therefore, $D$ is a function of frequency, permittivity, and the radius $R$. It can be increased by increasing any of the above parameters. Effectively this increases the electrical size of the insulating sphere, i.e., the electrical distance between the dipole element and the surface $S$. In implanted biomedical applications, $R$ is bounded by the overall 

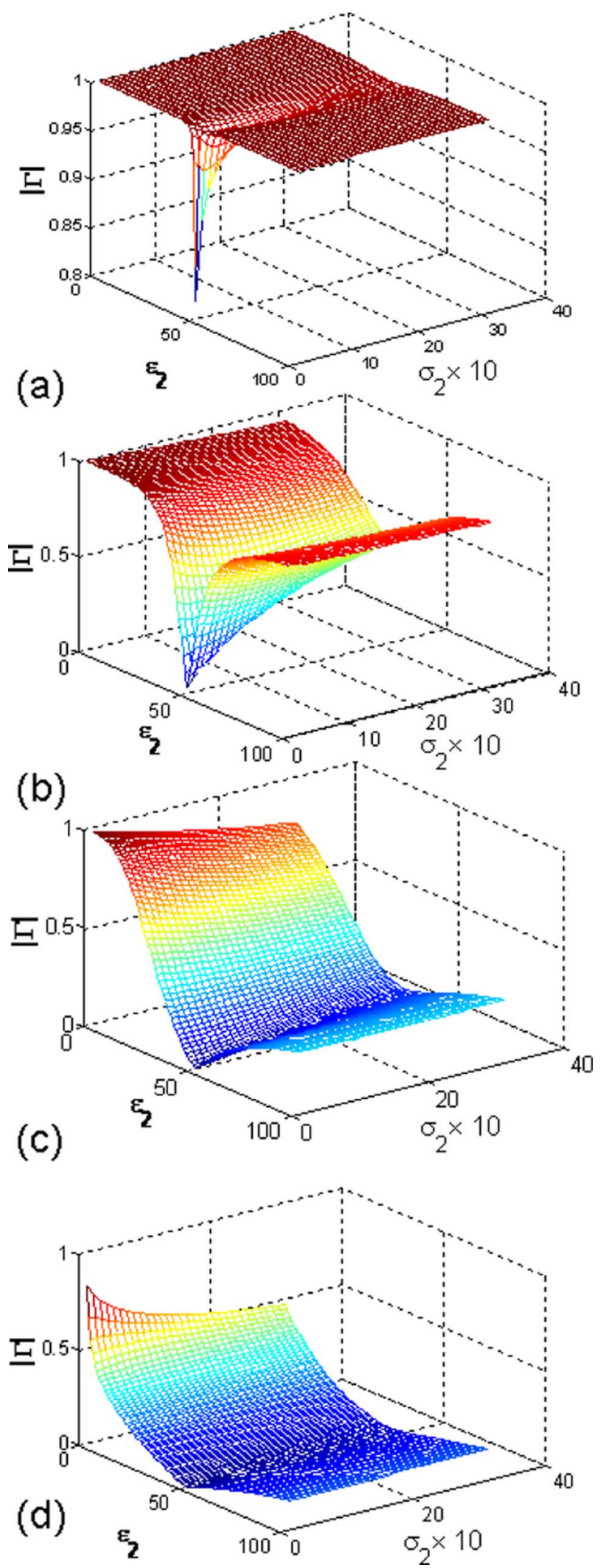

Fig. 2. Reflection coefficient magnitude at surface $S$ for: (a) $D=0.1$, (b) $D=$ 0.5 , (c) $D=1$, and (d) $D=5 . \varepsilon_{1}=50$. When $f=4 \mathrm{GHz}, R=$ $0.17,0.84,1.7$, and $8.4 \mathrm{~mm}$, respectively.

size of the device. To maximize $D$, which, in turn, minimizes the reflection coefficient, we shall select $\varepsilon_{1}$ as large as possible.
TABLE I

DiELECTRIC PROPERTIES OF VARIOUS TISSUES AT 1 AND $11 \mathrm{GHz}$

\begin{tabular}{|l|l|l|l|l|}
\hline \multirow{2}{*}{} & \multicolumn{2}{|c|}{$1 \mathrm{GHz}$} & \multicolumn{1}{c|}{$11 \mathrm{GHz}$} \\
\cline { 2 - 5 } & $\sigma_{2}(\mathbf{S} / \mathbf{m})$ & $\varepsilon_{2}$ & $\sigma_{2}(\mathbf{S} / \mathbf{m})$ & $\varepsilon_{2}$ \\
\hline Aorta & 0.72866 & 44.561 & 10.298 & 31.425 \\
\hline Blood & 1.5829 & 61.065 & 14.777 & 43.421 \\
\hline BodyFluid & 1.6673 & 68.875 & 17.414 & 56.002 \\
\hline Kidney & 1.4495 & 57.939 & 12.983 & 38.836 \\
\hline Muscle & 0.97819 & 54.811 & 12.083 & 41.419 \\
\hline Stomach & 1.2316 & 64.797 & 15.075 & 47.184 \\
\hline Heart & 1.2836 & 59.29 & 13.329 & 40.735 \\
\hline
\end{tabular}

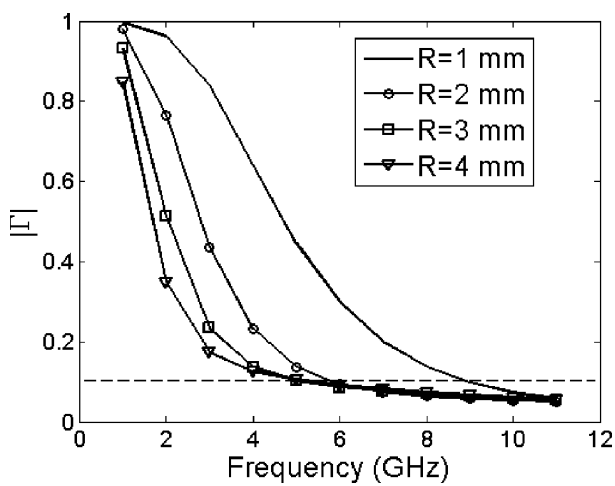

Fig. 3. Reflection coefficient against frequency for average permittivity and conductivity of tissue material.

In practice, however, this selection is governed by the properties of surrounding body tissue (because $\varepsilon_{1}=\varepsilon_{2}$ is the condition for minimum $|\Gamma|$ ) and availability of insulating material with suitable permittivity.

Further conditions are imposed by the frequency band of operation, as discussed in Section II-B.

\section{B. Dipoles in Body Tissue}

The average $\varepsilon_{2}$ for body tissue is around 50 within the industrial, scientific, and medical (ISM) 2.45-GHz band, with the exception of bones, bladder, and breast tissue [18]. Let us consider a wider frequency band of $1-11 \mathrm{GHz}$, which includes the full Federal Communications Commission (FCC) UWB frequency band. Dielectric properties of a selected set of tissue types at 1 and $11 \mathrm{GHz}$ are shown in Table I. We assumed the average electromagnetic properties of dissipative media over this band to be $\varepsilon_{2}=47, \sigma_{2}=7 \mathrm{~S} / \mathrm{m}$, as an example to cover across a wide range of tissue types, and used this constant value for simplicity. Let $\varepsilon_{1}=50$.

Fig. 3 shows the $\Gamma$ variation with frequency for those parametric values. As an example, $|\Gamma|=0.1$ has been chosen as the acceptable threshold reflection coefficient. It is obvious that the frequency at which the reflection coefficient reaches the threshold $|\Gamma|=0.1$ decreases with increasing $R$. In other words, for small spheres, low reflection coefficients are possible only at higher frequencies.

When frequency increases, the $|\Gamma|$ reaches an asymptotic value close to zero. Therefore, if low $|\Gamma|$ is achieved at the lower limit of the operating bandwidth, the antenna will not experience surface reflections [i.e., at $(S)$ ] for the rest of the operating frequencies, provided other conditions remain unchanged. 
TABLE II

APPROXIMATED BODY TISSUE PROPERTIES FOR SiMULATIONS AND CALCULATIONS

\begin{tabular}{|l|l|l|}
\hline Tissue Type & $\varepsilon_{2}$ & $\sigma_{2}(\mathbf{S} / \mathbf{m})$ \\
\hline Aorta/ Liver & 43 & 1.47 \\
\hline Bladder & 18 & 0.69 \\
\hline Blood & 58 & 2.58 \\
\hline Breast/fat & 5 & 0.14 \\
\hline Kidney/Muscle & 53 & 1.77 \\
\hline Small intestine & 54 & 3.21 \\
\hline Stomach & 62 & 2.25 \\
\hline
\end{tabular}

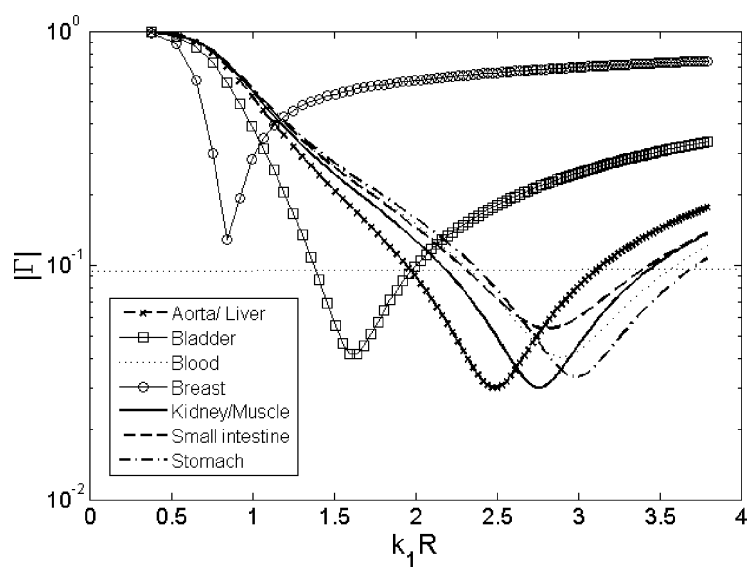

Fig. 4. Variation of the reflection coefficient at $S$ with the dielectric constant of the insulator, for various dissipative media. Frequency fixed at $4 \mathrm{GHz}$.

This is essentially the main challenge in UWB antenna miniaturization-reducing electrical dimensions of the antenna to meet the requirements at the lower end of the operating bandwidth. In this case, the solution is increasing the electrical distance between antenna current elements and the boundary surface between media.

Thus, the following conditions should be imposed when selecting the insulating material.

- The dielectric constant should be equal to or close to that of surrounding dissipative medium.

- $k_{1} R$ is maximized so that reflection coefficients are less sensitive to the variable properties of dissipative medium.

Note that the above requirements are not mutually exclusive. Once the reflections at $S$ (Fig. 1) are removed (or minimized) by selecting the insulating material based on above guidelines, conventional broadband antenna design techniques can be followed.

The approximations shown in Table II have been made for the properties of tissue material. Although these parameters are frequency dependent, we assumed them to be constant in the frequency range of consideration, i.e., $3.5-4.5 \mathrm{GHz}$ [18]. From 3 to $5 \mathrm{GHz}$, the dielectric constant reduces by less than $10 \%$. Fig. 4 shows $|\Gamma|$ against $k_{1} R$ for different tissue media surrounding an insulated Hertzian dipole at $4 \mathrm{GHz}$. Obviously, with the exemption of bladder and breast/fat tissue, across a wide range of $k_{1} R$, the reflection coefficient is less than $0.1(|\Gamma|<0.1)$. These correspond to high dielectric constants close to 50 for both insulation and tissue material. For lower dielectric constants, and thereby lower $k_{1} R$, even the minimum $|\Gamma|$ does not reach 0.1

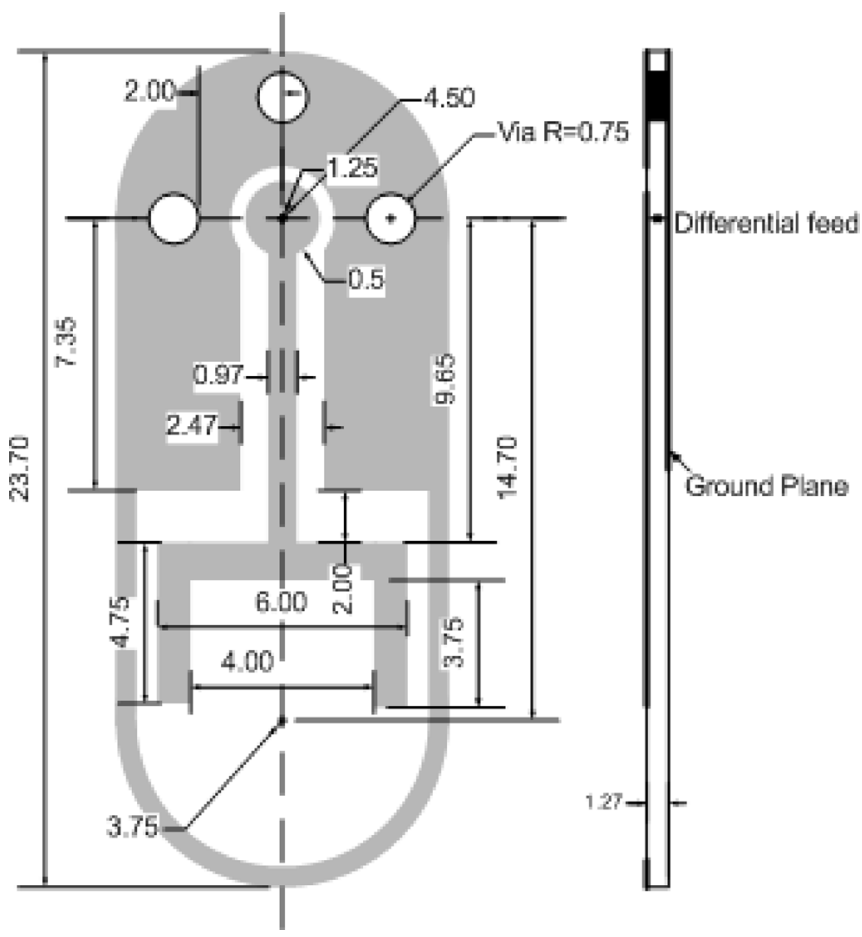

Fig. 5. Dimensions of the printed antenna on TMM substrate. Shaded area is metallization. Dimensions are in millimeters.

and the "matched" range is narrow. When the average dielectric constant of the tissue is in the range of 5-10, the dielectric constant of the insulator should be appropriately lowered.

The above knowledge has been obtained by considering a Hertzian dipole, due to its simplicity and availability of analytical solutions. However, Hertzian dipoles are not practical. In Section III, we apply this knowledge to design a practical magnetic-dipole-based UWB antenna, which is impedance matched across the selected wide frequency band.

\section{UWB ImPLANTEd ANTENNA SimULATIONS}

\section{A. Antenna Configuration}

Fig. 5 shows the dimensions of the new capsule-shaped dielectric-loaded implantable wide-slot antenna, which is printed on an RO TMM 10i substrate that has a dielectric constant of 9.8 and a loss tangent 0.002 . The substrate has the shape of a capsule and its dimensions are conformal to a longitudinal cross section of a size 000 capsule. The thickness of the substrate is $1.27 \mathrm{~mm}$. One side of the substrate is etched with a grounded coplanar waveguide (CPW) (with an additional ground plane on the opposite face of the substrate) and a wide-slot antenna with a U-shape feed, a UWB antenna configuration previously considered for operation in an air medium in other applications [15]. The three vias, each with a $0.75-\mathrm{mm}$ radius, connect the two ground planes on the opposite sides of the substrate. The feed in simulations is differential between the upper ground plane and the center of the circular pad at the start of the CPW. Circular pad arrangement facilitates a broadband probe-to-CPW transition [22]. This transition is useful when subminiature A (SMA) connector probes are used in prototypes. The slot precisely occupies the lower half of the substrate. This half is loaded with 


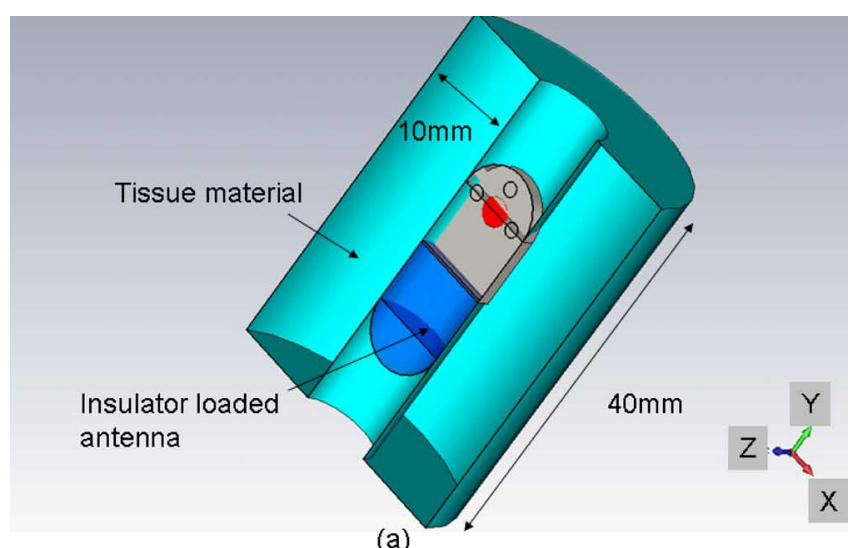

(a)

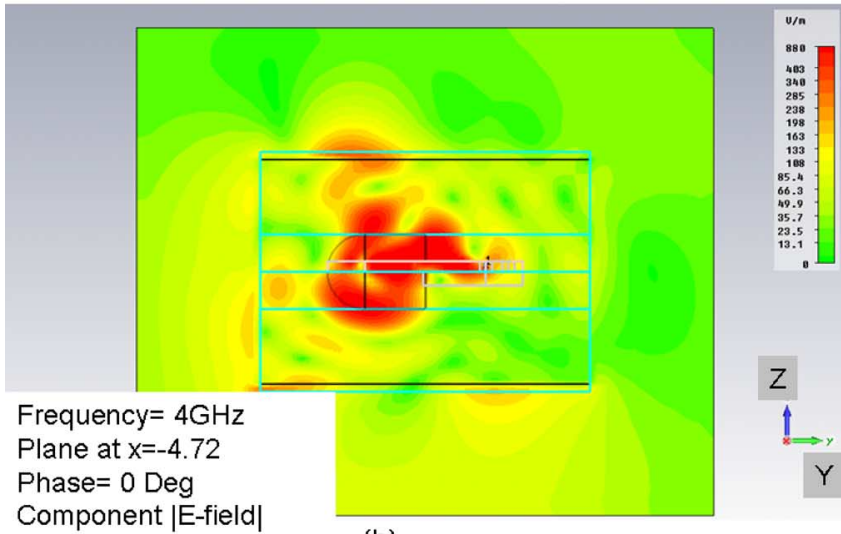

(b)

Fig. 6. Simulated antenna in a cylinder of tissue material. (a) Cross-sectional view. (b) Distribution of electric field magnitude. Open radiation boundaries $14 \mathrm{~mm}$ from the object sides.

glycerine as the insulating material, which has a dielectric constant of 50. Dielectric loss of glycerine is assumed negligible.

Shown in Fig. 6(a) is the simulated capsule-shaped antenna, loaded with glycerine, inside a cylinder of tissue material. The inner radius of tissue cylinder is $4.5 \mathrm{~mm}$, equal to the outer radius of the capsule. Considering the aim of the simulation, that is to investigate the matching of the antenna and the near-field effects, the cylinder thickness has been set to $10 \mathrm{~mm}$. Further increase of the cylinder thickness increases simulation time, but does not change $S 11$ values. Properties of the cylinder material are selected as given by Table II and simulations have been carried out using CST Microwave Studio from 3 to $5 \mathrm{GHz}$. The distribution of electric field magnitude at $4 \mathrm{GHz}$ is shown in Fig. 6(b). A strong near field is present inside the insulator, closer to the slot. Field strength outside the tissue simulating material, i.e., far field, is about eight times weaker.

Antenna input reflection coefficient is plotted in Fig. 7 for different tissue environments. Note that the results are directly correlated to those of Fig. 4. Except for breast and bladder, the antenna has a return loss greater than $10 \mathrm{~dB}$ in the frequency range of 3.5-4.5 GHz.

This frequency range is the operating bandwidth of our impulse-UWB test setup that has also been used in some recent implantable systems [23]. In the case of breast tissue, the matched band is narrow, corresponding to low $k_{1} R$, as expected from the characteristics shown in Fig. 4.

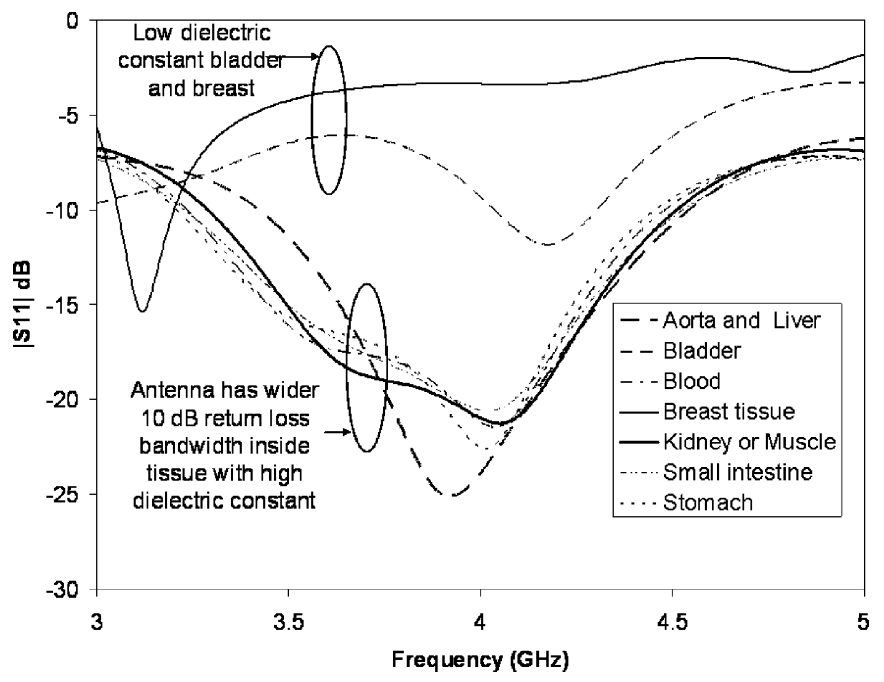

Fig. 7. Antenna input reflection coefficient, when the antenna is surrounded by different tissues.

TABLE III

RADIATION AND TOTAL EFFICIENCY OF THE SLOT ANTENNA IN Dissipative MEdia at $4 \mathrm{GHz}$

\begin{tabular}{|c|c|c|}
\hline $\begin{array}{c}\text { Tissue } \\
\text { Material }\end{array}$ & $\begin{array}{c}\text { Total } \\
\text { Efficiency }\end{array}$ & $\begin{array}{c}\text { Radiation } \\
\text { Efficiency }\end{array}$ \\
\hline Aorta/ Liver & 0.02 & 0.02 \\
\hline Bladder & 0.06 & 0.07 \\
\hline Blood & 0.01 & 0.01 \\
\hline Breast & 0.40 & 0.20 \\
\hline $\begin{array}{c}\text { Kidney/ } \\
\text { Muscle }\end{array}$ & 0.02 & 0.02 \\
\hline $\begin{array}{c}\text { Small } \\
\text { Intestine }\end{array}$ & 0.008 & 0.008 \\
\hline Stomach & 0.01 & 0.01 \\
\hline
\end{tabular}

\section{B. Transmission Efficiency}

The radiation efficiency $e_{\text {rad }}$ and the total efficiency $e_{\text {total }}$ are given by

$$
\begin{aligned}
& e_{\text {rad }}=\frac{P_{\text {out }}}{P_{\text {in }}-P_{\text {mismatch_loss }}} \text { and } e_{\text {total }}=\frac{P_{\text {out }}}{P_{\text {in }}} \\
& P_{\text {out }}=P_{\text {in }}-P_{\text {mismatch_loss }}-P_{\text {ant }}-P_{\text {tissue }}
\end{aligned}
$$

where $P_{\text {in }}$ is the power of the transmitter, $P_{\text {mismatch_loss }}$ is the power loss due to antenna-source mismatch, $P_{\text {ant }}$ is the internal copper and dielectric losses of the antenna, $P_{\text {tissue }}$ is the loss inside the tissue material, which is the most prominent, and $P_{\text {out }}$ is the total power received at the outside of the tissue cylinder. In our simulations, $P_{\text {ant }}$ is only due to dielectric losses in an RO TMM 10i substrate as we have assumed perfect electric conductors.

Table III shows the radiation efficiency $e_{\text {rad }}$ and the total efficiency $e_{\text {total }}$ given by (4) and (5). The losses in the tissue cylinder at $4 \mathrm{GHz}$ have been included when calculating both parameters. When $P_{\text {mismatch_loss }}$ is close to zero, from (4), $e_{\text {rad }}=$ $e_{\text {total }}$. In these simulations, it is obvious that $P_{\text {mismatch_loss }}$ can be reduced close to zero by better matching of the antenna. Almost all the losses are then in the tissue material. The gain values recorded in these simulations are within from -10 to $-16 \mathrm{dBi}$, 

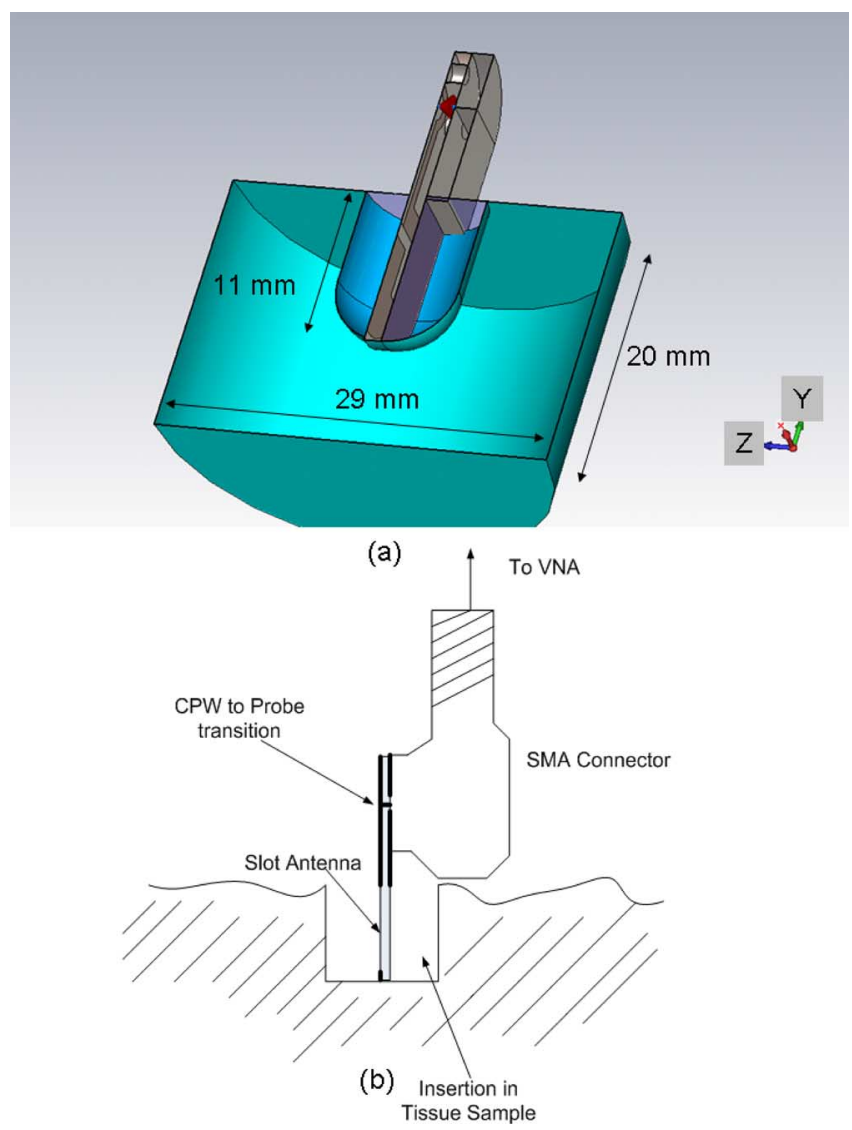

Fig. 8. Antenna and tissue sample. (a) Simulation. (b) Experiment set up. Open radiation boundaries $14 \mathrm{~mm}$ from the object sides.

comparable to maximum gain reported in [10] at a lower frequency and inside a 24-mm-thick block of tissue. This gain has been calculated for the combination of the antenna and the tissue material.

Within the UWB frequency band, the effective isotropic radiated power (EIRP) is restricted to $-41 \mathrm{dBm} / \mathrm{MHz}$ by regulations. With the overall gain values in the range from -10 to $-16 \mathrm{dBi}$, for this particular simulated scenario, the upper limit for transmitter output power spectral density (PSD) should be in the range from -31 to $-25 \mathrm{dBm} / \mathrm{MHz}$. Note that EIRP regulations are imposed in the free space. Provided safe specific absorption ratio (SAR) levels and battery consumption limits are met, transmitter PSD level can be set to the above values, and hence, the out-of-body UWB receiver will not require extra sensitivity.

\section{Measured Results}

A proof-of-concept experiment has been conducted with an antenna prototype. A right-angle SMA connector is soldered to the antenna printed circuit board, as shown in Fig. 8(b). A photograph of the antenna prototype is shown in the lower right corner of Fig. 9. The antenna input reflection coefficients were measured using an E5071B calibrated vector network analyzer. As shown in Fig. 9, the cable and antenna were held above samples of pork midlion chops using a holding stand. In the experiment, the antenna has been partially embedded.

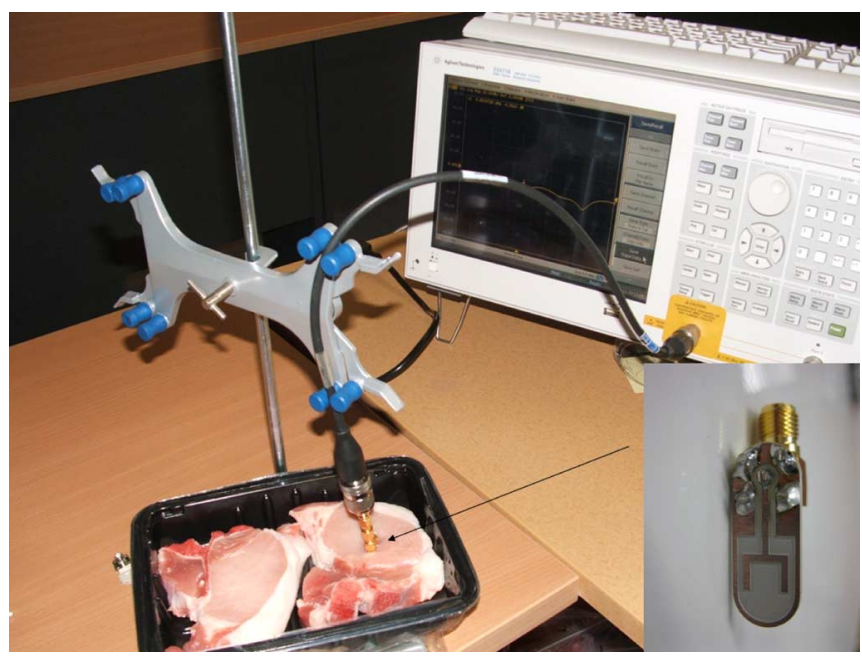

Fig. 9. Photograph of the measurement setup including the VNA.

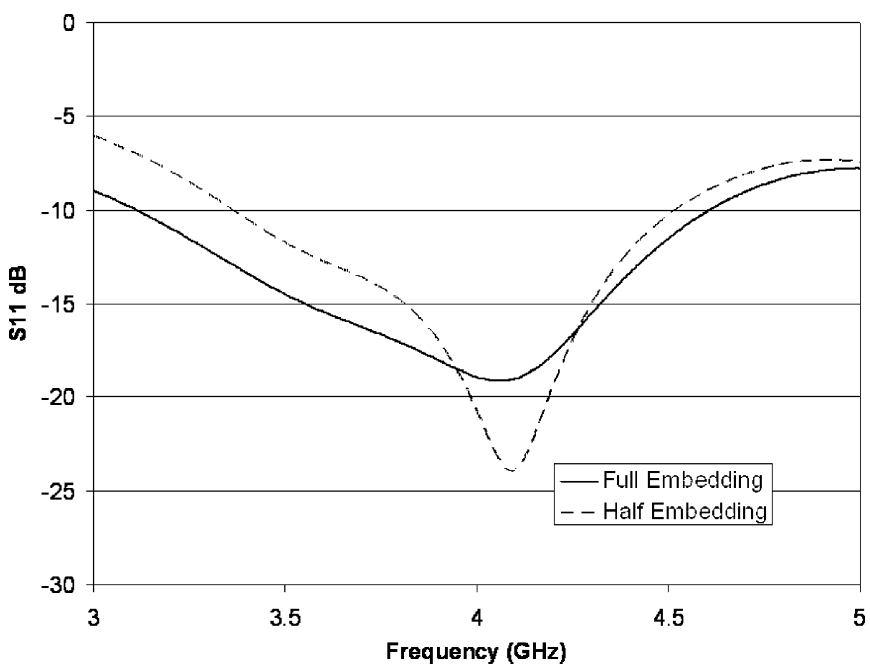

Fig. 10. Simulated input $\left|S_{11}\right|$ for full and partial embedding of the antenna in tissue material.

The theoretical antenna input reflection coefficients for both partial and full embedding of the antenna are shown in Fig. 10. In partial embedding simulation, the antenna penetration is $11 \mathrm{~mm}$ and the height of the dielectric cylinder has been reduced to $20 \mathrm{~mm}$, to cover the loaded portion of the antenna, as shown in Fig. 8(a). The electromagnetic properties of the insulator and tissue cylinder remained the same in both simulations: $\varepsilon_{1}=50, \varepsilon_{2}=47, \sigma_{2}=7 \mathrm{~S} / \mathrm{m}$. According to Fig. 10, at most a 5-dB difference between $S_{11}$ values can be expected due to the differences in embedding. Although we can see close to 250-MHz reduction from the lower end of the 10- $\mathrm{dB}$ return-loss bandwidth due to partial embedding, the $S_{11}$ curve profile is the same for both cases.

On two samples of pork chops, of which dielectric properties were assumed to be close to that of human tissue, insertions were carved out using a sharp knife and they were moulded using a size 000 gelatine capsule. (The capsule was not present during the experiments, as they dissolve due to moisture.) Depth of these insertions is approximately $11 \mathrm{~mm}$, sufficient to cover the 


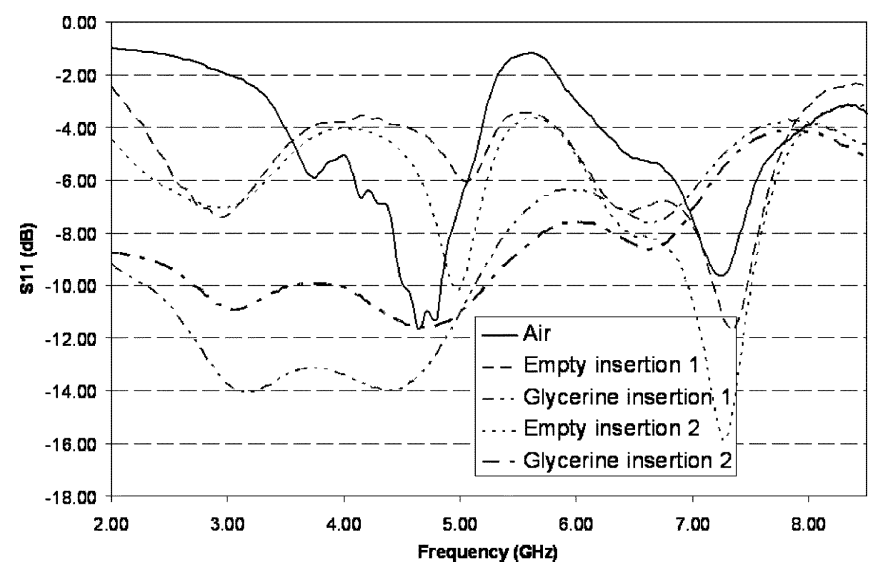

Fig. 11. Measured input $\left|S_{11}\right|$ from the proof-of-concept experiment.

slot portion of the printed antenna. First the antenna input $S_{11}$ was measured while it was freely suspending in space. The antenna was then lowered into the insertion and $S_{11}$ was measured again. Finally, the insertion was carefully filled with Glycerine using a dropper. Measured $S_{11}$ values for both insertions (insertion 1 and insertion 2) are shown in Fig. 11.

In free space, as expected, this antenna does not behave as a UWB antenna at all. There are two resonances visible around 4.5 and $7.25 \mathrm{GHz}$. The former is quite expected considering the electrical size of the antenna prior to loading. These two resonances are also present when the antenna is measured inside empty insertions. As can be seen in Fig. 11, the filling of insertions with Glycerine has a significant effect. In this case, the antenna is matched in the $3-5-\mathrm{GHz}$ frequency band with a return loss greater than $10 \mathrm{~dB}$. The simulated $S 11$ in Fig. 10 does not agree well with the measured results in Fig. 11. This is primarily due to not knowing the exact electrical properties of the pork sample and glycerine sample. In addition, the pork sample is not as homogenous as modeled in simulations. The 3-dB difference in return loss between two insertions is further evidence to that. Nevertheless clear improvement in $S_{11}$ in the operating band, due to insulator loading, is obvious.

\section{CONCLUSION}

An effective method to achieve impedance matching of UWB implanted antennas with dielectric loading has been studied. The dielectric constant of insulated material should be selected close to that of external dissipative medium, as shown theoretically using a Hertzian dipole in a dissipative medium. We have also shown that when the electrical distance between the current elements and the insulator-tissue boundary is increased, acceptable low reflection levels can be sustained across a wide range of frequencies or a wide range of tissue properties. When it is not possible to extend the physical radius of the insulator, there are two other options.

One is to increase the dielectric constant. According to the results presented here, when the dielectric constants involved are in the vicinity of 40-50, wideband matching of the antenna can be achieved. This is encouraging as most of the tissue materials have high dielectric constants, close to 50 , at least in the lower
UWB band. Therefore, glycerine, which has a dielectric constant of about 50 and very low dielectric losses, is a suitable insulator material. The second is to move toward higher operating frequencies. However, this also requires improved receiver sensitivity to counter higher tissue losses at high frequencies.

An experiment conducted with a glycerine-loaded UWB capsule-shaped antenna, designed using CST MWS, has verified the new concept of antenna design. With proper enclosure and extra packaging techniques, it is possible to modify the proposed antenna for different practical implants. The effect of low dielectric-constant biocompatible encasing on the performance of the antenna can be analyzed with a similar approach that has been followed in [17].

\section{REFERENCES}

[1] T. Karacolak, A. Z. Hood, and E. Topsakal, "Design of a dual-band implantable antenna and development of skin mimicing gels for continuous glucose monitoring," IEEE Trans. Microw. Theory Tech., vol. 56, no. 4, pp. 1008-1008, Apr. 2008.

[2] J. Kim and Y. Rahmat-Samii, Implanted Antennas in Medical Wireless Communications, 1st ed. San Rafael, CA: Morgan \& Claypool, 2006.

[3] S. Soora, K. Gosalia, M. S. Humayan, and G. Lazzi, "A comparison of two and three dimensional dipole antennas for an implantable retinal prosthesis," IEEE Trans. Antennas Propag., vol. 56, no. 3, pp. 622-629, Mar. 2008

[4] J. Ryckaert, C. Desset, A. Fort, M. Badaroglu, V. De Heyn, P. Wanbacq, G. Van der Plas, S. Donnay, B. Van Poucke, and B. Gyselinckx, "Ultra-wide-band transmitter for low-power wireless body area networks: Design and evaluation," IEEE Trans. Circuits Syst. I, Reg. Papers, vol. 52, no. 12, pp. 2515-2525, Dec. 2005.

[5] H. Yu, G. S. Irby, D. M. Peterson, M. T. Nguyen, G. Flores, N. Euliano, and R. Bashirullah, "Printed capsule antenna for medication compliance monitoring," Electron. Lett., vol. 43, no. 22, pp. 41-44, 2007.

[6] C. Gabriel, Compilation of the Dielectric Properties of Body Tissues at RF and Microwave Frequencies. London, U.K.: King's College, 1996.

[7] S. K. S. Gupta, S. Lalwani, Y. E. E. Prakash, and L. Schwiebert, "Towards a propagation model for wireless biomedical applications," in IEEE Int. Commun. Conf., May 2003, vol. 3, pp. 1993-1997.

[8] L. C. Chirva, P. A. Hammond, S. Roy, and D. R. S. Cumming, "Radiation from ingested wireless devices in biomedical telemetry bands," Electron. Lett., vol. 39, no. 2, pp. 178-179, Jan. 2003.

[9] P. Soontornpipit, C. M. Eurse, and Y. C. Chung, "Design of implantable microstrip antenna for communication with medical implants," IEEE Trans. Microw. Theory Tech., vol. 52, no. 8, pp. 1944-1955, Aug. 2004.

[10] C.-M. Lee, T.-C. Yo, and C.-H. Luo, "Compact broadband stacked implantable antenna for biotelemetry with medical devices," in IEEE Annu. Wireless Microw. Technol. Conf., Dec. 4-5, 2006, pp. 1-4.

[11] R. Warty, M. Tofighi, R. U. Kawoos, and A. Rosen, "Characterization of implantable antennas for intracranial pressure monitoring: Reflection by and transmission through a scalp phantom," IEEE Trans. Microw. Theory Tech., vol. 56, no. 10, pp. 2366-2376, Oct. 2008.

[12] A. Djaiz, M. Nedil, M. A. Habib, L. Talbi, and T. A. Denidni, "A new CPW-feed miniaturized dual-band monopole antenna using spiral matching resonators," in IEEE Int. Antennas Propag. Symp., San Diego, CA, 2008, pp. 1-4.

[13] M. R. Yuce, W. Liu, M. S. Chae, and J. S. Kim, "A wideband telemetry unit for multi-channel neural recording systems," in IEEE Int. UltraWideband Conf., Sep. 2007, pp. 612-617.

[14] T. Dissanayake, M. R. Yuce, and C. Ho, "Design and evaluation of a compact antenna for implant-to-air UWB communication," IEEE Antennas Wireless Propag. Lett., vol. 8, pp. 153-156, 2009.

[15] T. Dissanayake and K. P. Esselle, "UWB performance of compact L-shaped wide slot antennas," IEEE Trans. Antennas Propag., vol. 56, no. 4, pp. 1183-1187, Apr. 2008.

[16] H. G. Schantz, "UWB magnetic antennas," in IEEE Int. Antennas Propag. Symp., 2003, vol. 3, pp. 604-607. 
[17] C. T. Tai and R. E. Collin, "Radiation of a Hertzian dipole immersed in a dissipative medium," IEEE Trans. Antennas Propag., vol. 48, no. 10, pp. 1501-1506, Oct. 2000.

[18] P. S. Hall and Y. Hao, Antennas and Propagation for Body-Centric Wireless Communications. Norwood, MA: Artech House, 2006.

[19] S. M. Mikki and A. K. Kishk, "Theory and applications of infinitesimal dipole models for computational electromagnetics," IEEE Trans. Antennas Propag., vol. 55, no. 5, pp. 1325-1337, May 2007.

[20] E. K. Miller and J. A. Landt, "Direct time-domain techniques for transient radiation and scattering from wires," Proc. IEEE, vol. 68, no. 11, pp. 1396-1423, Nov. 1980.

[21] R. G. Martin, A. R. Bretones, and S. G. Garcia, "Some thoughts about transient radiation by straight thin wires," IEEE Antennas Propag. Mag., vol. 41, no. 3, pp. 24-33, 1999.

[22] T. Kamei, Y. Utsumi, N. Q. Dinh, and N. Thanh, "Wide-band coaxial-to-coplanar transition," IEICE Trans. Electron., vol. E90-C, no. 10, pp. 2030-2036, 2007.

[23] M. Chae, W. Liu, Z. Yang, T. Chen, J. Kim, M. Sivaprakasam, and M. R. Yuce, "A 128-channel $6 \mathrm{~mW}$ wireless neural recording IC with on-the-fly spike sorting and UWB transmitter," in IEEE Int. Solid-State Circuits Conf., 2008, pp. 312-321.

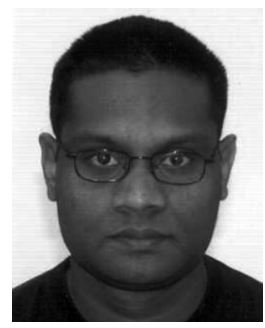

Tharaka Dissanayake (S'02-M'07) received the B.Sc.Eng. degree in electronics and telecommunications engineering (with first-class hons.) from the University of Moratuwa, Moratuwa, Sri Lanka, in 2002, and the Ph.D. degree in electronics from Macquarie University, Sydney, N.S.W., Australia, in 2007.

From 2002 to 2003, he was an Assistant Lecturer with the University of Moratuwa. He has been a Visiting Lecturer with the Kotalawala Defence Academy and Arthur C. Clerk Centre for Modern Technology. He was also a Lecturer in electronics with Macquarie University. He is currently with the School of Electrical Engineering and Computer Science, University of Newcastle, Newcastle, N.S.W., Australia. His current research interests are in UWB antennas, multiple-input multiple-output (MIMO) systems, microwave imaging techniques, and wireless power transfer.

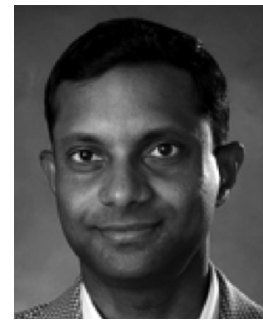

Karu P. Esselle (M'92-SM'96) was the Associate Dean-Higher Degree Research, Member of the Division Executive, and the Founding Director of the Postgraduate Research Committee, Division of Information and Communication Sciences, Macquarie University, Sydney, N.S.W., Australia from 2002 to 2008 . He is currently the Director of the Centre for Electromagnetic and Antenna Engineering, and the Deputy Director of the Research Centre on Microwave and Wireless Applications, Macquarie University, recently funded as a Concentration of Research Excellence. Since 2002, he has been involved with research grants and contracts worth approximately 4.4 million dollars, and his research team members have attracted further grants worth approximately a million dollars. $\mathrm{He}$ has authored over 230 scientific publications, including five invited book chapters and over 15 invited conference presentations. He has been invited to serve as an international expert/ research grant assessor by overseas research grant organizations in The Netherlands, Finland, Hong Kong, Chile, etc. In Australia, he has been invited to assess grant applications submitted to the nation's most prestigious schemes such as Australian Federation Fellowships and Australian Laureate Fellowships. His industry experience includes full-time employment as a Design Expert with the Hewlett-Packard Company and several consultancies for local and international companies including Cisco, Optus, Locata/QX, ResMed, FundEd and Peter-Maxwell.

Prof. Esselle was the recipient of Macquarie University's first ever Vice Chancellor's Award for Excellence in Higher Degree Research Supervision in 2008 and the 2004 Macquarie University (Inaugural) Innovation Award for the best invention disclose.

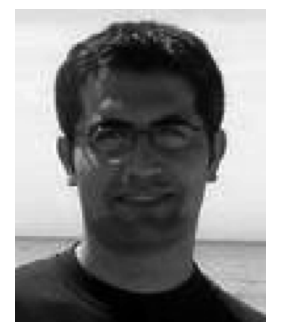

Mehmet R. Yuce (S'01-M'05) received the B.S. degree in electronics engineering from Ankara University, Ankara, Turkey, in 1997, the M.S. degree in electrical and computer engineering from the University of Florida, Gainesville, in 2001, and the Ph.D. degree from North Carolina State University (NCSU), Raleigh, in 2004.

From August 2001 to October 2004, he was as a Research Assistant with the Department of Electrical and Computer Engineering, NCSU. In 2005, he was a Post-Doctoral Researcher with the Electrical Engineering Department, University of California at Santa Cruz. He is currently an academic member with the Department of Electrical Engineering and Computer Science, University of Newcastle, Callaghan, N.S.W., Australia. His research interests include analog/digital mixed-signal very large scale integration (VLSI) for wireless, biomedical, and RF applications, wireless sensor network for medical monitoring, low-power VLSI circuits for emerging technologies in wireless communications, CMOS transceiver design, and system designs for wireless communications. 